\title{
FINITE ELEMENT ANALYSIS OF A SUPERELASTIC SHAPE MEMORY ALLOY CONSIDERING THE EFFECT OF PLASTICITY
}

\author{
XiAngJun JiAnG \\ Xidian University, Key Laboratory of Electronic Equipment Structural Design, Xi'an, China \\ State Key Laboratory of Structural Analysis for Industrial Equipment, Dalian University of Technology, Dalian, China \\ State Key Laboratory for Manufacturing Systems Engineering, Xi'an Jiaotong University, Xi'an, China \\ BAOTONG LI \\ Xi'an Jiaotong University, State Key Laboratory for Manufacturing System, Xi'an, China \\ e-mail: baotong.me@mail.xjtu.edu.cn (corresponding author)
}

\begin{abstract}
In the present study, a shape memory alloy (SMA) phenomenological constitutive model is proposed that is capable of describing SMA superelastic behavior and the plasticity effect. The phase transformation constitutive model, by using strain and temperature as control variables to judge the phase transformation points in order to avoid the complexity of transformation correction, incorporates plasticity described by the von Mises isotropic hardening model. Further, the proposed model is implemented into the finite element package ANSYS by the user subroutine USERMAT. The results produced by the proposed model of simulated superelastic and plasticity behavior are compared with experimental data taken from the literature.
\end{abstract}

Keywords: SMAs, superelasticity, plasticity, constitutive law, finite element analysis

\section{Introduction}

SMA is a kind of functional material which has the function of sensing and driving. It has been applied to many fields because of its shape memory, super elasticity and so on. Until recently, there are very few constitutive models suitable to consider both of stress-induced martensite transformation and plastic deformation of martensite. McKelvey and Ritchie (2001) found that plastic strain of the stress-induced martensite phase occurred if the applied stress was high enough. Lazghab (2001) carried out a theoretical study to SMAs with plastic deformation of martensite, although it was not available to implement into a commercial finite element software. Yan et al. (2003) developed a constitutive model to quantify the effect of plasticity on the reverse transformation and examined the influence of hydrostatic stress on the transformation. Bo and Lagoudas (1999) proposed several constitutive models considering the evolution of plastic strain under cyclic thermal induced transformation cycles with micromechanical analyses by a representative volume element. However, those models cannot be utilized to analyze the combination of super-elastic and plastic behavior due to their limitations in the description of martensite plasticity.

Some works have been carried out to implement the super-elastic constitutive model into the finite element model (Auricchio and Taylor, 1997). The super-elastic constitutive model proposed by Auricchio and Taylor (1997) has been successfully implemented into finite element codes such as ABAQUS and ANSYS. However, that model cannot describe plasticity of the martensite. In a more recent work, Kan et al. (2010) and Yan et al. (2003) developed a temperature-dependent three-dimensional phenomenological constitutive model considering the local plastic yield of martensite under a high stress, and successfully implemented into the finite 
element package ABAQUS. Moreover, the nonlinear problem was solved within a framework of the updated Lagrangian formulation using a return mapping algorithm (Lagoudas et al., 1996; Cisse et al., 2016) to update the stress value according to the current strain. However, the phase transformation starting conditions in that model using the phase diagram $(\sigma, T)$ to determine the SMA phase state (Bouvet et al., 2004; Saint et al., 2009) is complex and not suitable for identifying.

In the present work, a modified constitutive model having temperature and the strain tensor as control variables, is developed. It analyzes thermomechanical behavior of 3D SMA structures with a feature of combination of superelasticity and plasticity, which is particularly suitable for finite element implementation. Moreover, the finite element analysis of a SMA structure considers nonlinearities associated with phase transformation and plasticity. The modified constitutive model is particularly suitable for identifying the starting conditions of phase transformation and plasticity, and for finite element implementation. However, some other phenomena observed in SMAs including re-orientation of the martensite phase and a non-linear transformation hardening function are not taken into account in the construction of the constitutive model.

\section{Constitutive model}

\subsection{Thermodynamic state equation and internal variable}

The constitutive model in this work will be constructed under the generalized plasticity theory. Lubliner and Auricchio applied this theory to study SMAs, and proposed a three-dimensional thermo-dynamic constitutive model which can be used to simulate the effect of super elasticity and shape memory effect of SMA (Lubliner and Auricchio, 1996). The following is a brief description of the generalized plastic theory.

On the infinitesimal strain assumption, the additively decomposition of the total strain $\varepsilon$ into an elastic strain $\varepsilon_{e}$ and an inelastic strain $\varepsilon_{i n}$ yields

$$
\varepsilon=\varepsilon_{e}+\varepsilon_{i n}
$$

It is assumed that the Helmoltz free energy can also be additively contributed to elastic and inelastic parts as follows

$$
\boldsymbol{\psi}=\boldsymbol{\psi}_{e}\left(\varepsilon-\varepsilon^{i n}, \xi, T\right)+\boldsymbol{\psi}_{i n}(\xi, \eta, T)
$$

in which $\xi$ and $\eta$ depict phase transformation and plastic yield behavior, respectively.

The elastic free energy $\boldsymbol{\psi}_{e}$ is different from that of the classical elastic, plastic or visco-elastic theory, which is related to the internal variable $\xi$, and is shown as follows

$$
\boldsymbol{\psi}_{e}=\frac{1}{2}\left(\varepsilon-\varepsilon^{i n}\right): \mathbf{D}(\xi):\left(\varepsilon-\varepsilon^{i n}\right)
$$

in which $\mathbf{D}(\xi)$ is the elastic stiffness matrix.

The stress can be derived from the thermodynamic state equation

$$
\boldsymbol{\sigma}=\frac{\partial \psi_{e}}{\partial \varepsilon^{e}}=\mathbf{D}(\xi):\left(\varepsilon-\varepsilon^{i n}\right)
$$

The above process can be directly extended into construction of the constitutive model of the SMA. 


\subsection{Main equations of the constitutive model}

Based on the assumption of small deformation, the total inelastic strain $\varepsilon_{i n}$ can be divided into two parts: stress-induced martensitic transformation $\varepsilon_{t}$ and irreversible plastic strain $\varepsilon_{p}$, in which $\varepsilon_{p}$ includes austenite plastic strain $\varepsilon_{p}^{A}$ in a high temperature and martensite plastic strain $\varepsilon_{p}^{M}$ under a high stress. As a result, the total strain is

$$
\varepsilon=\varepsilon_{e}+\varepsilon_{i n}=\varepsilon_{e}+\varepsilon_{t}+\varepsilon_{p}
$$

in which $\varepsilon_{p}=\varepsilon_{p}^{A}+\varepsilon_{p}^{M}$.

After discretizing the equations, the expressions are as follows

$$
\Delta \varepsilon_{i n}=\Delta \varepsilon_{t}+\Delta \varepsilon_{p} \quad \Delta \varepsilon_{p}=\Delta \varepsilon_{A p}+\Delta \varepsilon_{M p} \quad \Delta \varepsilon_{e}=\Delta \varepsilon-\Delta \varepsilon_{t}-\Delta \varepsilon_{p}
$$

For the super-elastic NiTi alloy, the stress-induced martensitic transformation and its reverse transformation can be expressed by the volume fraction of martensite, which can be defined by the internal variable $\xi$, so the elastic stress-strain relation can be expressed as

$$
\boldsymbol{\sigma}=\boldsymbol{\sigma}^{*}-\mathbf{D}^{e}(\xi): \Delta \varepsilon_{i n}
$$

in which $\boldsymbol{\sigma}^{*}=\mathbf{D}^{e}(\xi): \Delta \varepsilon$ is the trial stress, and $\mathbf{D}^{e}(\xi)$ is the equivalent elastic tensor. It can be changed with a change of the phase transformation, which is different than in general metal materials, from the initial austenite to the final martensite.

The simple form for the equivalent elastic tensor as $\mathbf{D}=\mathbf{D}_{A}=\mathbf{D}_{M}$ has been used in some researches because of its close proximity to the real elastic modulus of austenite and martensite (Tanaka, 1986; Liang and Rogers, 1990). In this paper, a scheme describing the equivalent elastic tensor is proposed to simplify the calculation as

$$
\mathbf{D}^{e}(\xi)=\frac{1}{2}\left(\mathbf{D}_{A}+\mathbf{D}_{M}\right)
$$

in which $\mathbf{D}_{A}$ and $\mathbf{D}_{M}$ are the elastic tensors of austenite and martensite, respectively. The experiment demonstrated that the effect of the difference between the elastic modulus of austenite and martensite could be ignored.

Metal materials are generally irrecoverably plastic. Their characteristics are independent of hydrostatic pressure. Therefore, the plastic yield surface can only consider the stress tensor. Considering the deviatoric stress of the above equation, we get $\mathbf{D}^{e}: \Delta \varepsilon_{i n}=2 G \Delta \varepsilon_{i n}$, and

$$
\mathbf{s}=\mathbf{s}^{*}-2 G \Delta \varepsilon_{i n}
$$

in which $\mathbf{s}^{*}$ is the deviatoric stress of $\boldsymbol{\sigma}^{*}, G=E /(1+\nu)$ is the shear elastic modulus in which $\nu$ is the Poisson ratio, and $E$ is the equivalent elastic modulus expressed as $E=\left(E_{A}+E_{M}\right) / 2$. Therefore, the shear elastic modulus $\mathrm{G}$ is independent of the volume fraction of martensite.

\subsection{Evolution rule of phase transformation strain}

The isotropic behavior of metal materials can be described by the von Mises yield surface. The expression of the yield behavior is as follows

$$
F(\boldsymbol{\sigma}, q)=\sigma_{e q}-\sigma_{y}(q)=0
$$

in which $\sigma_{e q}=\left[\frac{3}{2} \mathbf{s}: \mathbf{s}\right]^{1 / 2}$ is the von Mises equivalent stress, $\sigma_{y}$ is the monotonic tensile plastic yield stress of the metal material, $q$ is the cumulative plastic strain.

Similarly to the plastic yield surface of a metal material, the phase transformation function to describe the forward transformation and the reverse transformation are introduced as follows: 
— forward phase transformation

$$
F_{A M}(\boldsymbol{\sigma}, \xi)=\sigma_{e q}-\sigma_{s, T}^{A M}(\xi)=0
$$

— reverse phase transformation

$$
F_{M A}(\boldsymbol{\sigma}, \xi)=\sigma_{e q}-\sigma_{s, T}^{M A}(\xi)=0
$$

where $\sigma_{s, T}^{A M}=\sigma_{s_{0}, T}^{A M}+h_{A M} \varepsilon_{L} \xi$ and $\sigma_{s, T}^{M A}=\sigma_{s_{0}, T}^{M A}+h_{M A} \varepsilon_{L} \xi$ are the initial stresses of the forward phase transformation and the reverse phase transformation, respectively. $\sigma_{s_{0}, T}^{A M}$ and $\sigma_{s_{0}, T}^{M A}$ are the initial stress of the forward phase transformation and the reverse phase transformation, respectively. $h_{A M}$ and $h_{M A}$ are the hardening modulus of the martensitic transformation and the reverse transformation, respectively.

In this paper, the volume fraction of martensite is

$$
\xi=\frac{\bar{\varepsilon}_{t}}{\varepsilon_{L}}
$$

in which $\varepsilon_{L}$ is the maximum phase transformation strain under uniaxial tension, which can be determined by experimental results under monotonic loading and unloading. $\bar{\varepsilon}_{t}=\left[\frac{2}{3} \varepsilon_{t}: \varepsilon_{t}\right]^{1 / 2}$ is the equivalent phase transformation strain. When the phase transformation does not occur, the equivalent transformation strain is zero. This means that the volume fraction of martensite is $\xi=0$. With an increase in the equivalent transformation strain, the martensite volume fraction increases linearly and eventually the maximum value $\xi=1$ is achieved.

Equation (2.13) shows that the phase transformation strain is proportional to the volume fraction of martensite. As a result, the phase transformation strain rate can be given in the form similar to the plastic theory:

— forward phase transformation

$$
\dot{\varepsilon}_{t}=\dot{\varepsilon}_{t}^{A M}=\dot{\lambda}_{A M} \frac{\partial F_{A M}(\boldsymbol{\sigma}, \xi)}{\partial \boldsymbol{\sigma}}=\sqrt{\frac{3}{2}} \varepsilon_{L} \dot{\xi}_{\mathbf{n}_{A M}} \quad \dot{\xi}>0
$$

- reverse martensitic transformation

$$
\dot{\varepsilon}_{t}=\dot{\varepsilon}_{t}^{M A}=\dot{\lambda}_{M A} \frac{\partial F_{M A}(\boldsymbol{\sigma}, \xi)}{\partial \boldsymbol{\sigma}}=\sqrt{\frac{3}{2}} \varepsilon_{L} \dot{\xi} \mathbf{n}_{M A} \quad \dot{\xi}<0
$$

$\lambda_{A M}$ and $\lambda_{M A}$ are the transformation multipliers of the martensitic transformation and the reverse transformation, respectively (similar to the plastic multiplier in the plastic theory). $\mathbf{n}_{A M}$ and $\mathbf{n}_{M A}$ are the direction vectors of the forward and reverse phase transformation, respectively

$$
\mathbf{n}_{A M}=\frac{\partial F_{A M}(\boldsymbol{\sigma}, \xi)}{\partial \boldsymbol{\sigma}}=\sqrt{\frac{3}{2}} \frac{\mathbf{s}}{\sigma_{e q}} \quad \mathbf{n}_{M A}=\frac{\partial F_{M A}(\boldsymbol{\sigma}, \xi)}{\partial \boldsymbol{\sigma}}=\sqrt{\frac{3}{2}} \frac{\mathbf{s}}{\sigma_{e q}}
$$

In this paper, the phase transformation, austenite and martensite yield behavior under different temperature are considered in the process of implicit solution of the stress integral. In general, the plastic strain produced during the phase transformation ratcheting under cyclic loadings should not be ignored regarding the experiments by Kang et al. (2009). However, it is assumed that there is no interaction between them in a single cycle of loading and unloading. The stress-induced martensitic transformation and the plastic behavior of austenite and martensite are independent of each other. The plastic behavior is not considered in the course of implicit stress integration of the phase transformation in our research. The isotropic elastic-plastic constitutive model with the implicit stress integration method is extended to that method of the constitutive modeling of SMA. The transformation strain increment and the plastic strain 
increment are calculated by the backward Euler difference method respectively, and the transformation strain and the plastic strain are updated further.

According to the above assumptions, Eq. (2.9) can be further expressed as follows for the implicit stress integration process of the phase change behavior

$$
\mathbf{s}=\mathbf{s}^{*}-2 G \Delta \varepsilon_{t}
$$

By Eqs. (2.16), it can be obtained:

- forward phase transformation

$$
\Delta \varepsilon_{t}^{A M}=\sqrt{\frac{3}{2}} \varepsilon_{L} \Delta \xi \mathbf{n}_{A M} \quad \Delta \xi>0
$$

- reverse phase transformation

$$
\Delta \varepsilon_{t}^{M A}=\sqrt{\frac{3}{2}} \varepsilon_{L} \Delta \xi \mathbf{n}_{M A} \quad \Delta \xi<0
$$

in which

$$
\mathbf{n}_{A M}=\mathbf{n}_{M A}=\mathbf{n}_{t}=\sqrt{\frac{3}{2}} \frac{\mathbf{s}}{\sigma_{e q}}
$$

Substituting Eqs. (2.18) and (2.19) into Eq. (2.17), we obtain

$$
\mathbf{s}=\mathbf{s}^{*}-3 G \varepsilon_{L} \Delta \xi \frac{\mathbf{s}}{\sigma_{e q}}
$$

It is further converted to

$$
\mathbf{s}\left(1+3 G \frac{\varepsilon_{L} \Delta \xi}{\sigma_{e q}}\right)=\mathbf{s}^{*}
$$

If we take the contracted tensor product of each side of this with itself, we obtain

$$
\left(1+3 G \frac{\varepsilon_{L} \Delta \xi}{\sigma_{e q}}\right)^{2} \mathbf{s}: \mathbf{s}=\mathbf{s}^{*}: \mathbf{s}^{*}
$$

Defining $\sigma_{e q}^{*}=\left[\frac{3}{2} \mathbf{s}^{*}: \mathbf{s}^{*}\right]^{1 / 2}$ as the equivalent stress, Eq. (2.23) can be further transformed into

$$
\left(1+3 G \frac{\varepsilon_{L} \Delta \xi}{\sigma_{e q}}\right) \sigma_{e q}=\sigma_{e q}^{*} \quad \text { or } \quad \sigma_{e q}+3 G \varepsilon_{L} \Delta \xi=\sigma_{e q}^{*}
$$

This is a non-linear equation in $\Delta \xi$ which may be solved by the Newton-Raphson iterative method. Therefore, the martensite volume fraction increment is given as

$$
\mathrm{d} \Delta \xi=\frac{\sigma_{e q}^{*}-3 G \varepsilon_{L} \Delta \xi-r-\sigma_{y}}{3 G \varepsilon_{L}+h \varepsilon_{L}}
$$

in which $\sigma_{y}$ is the initial stress of the forward phase transformation $\sigma_{s, T_{0}}^{A M}$ or the reverse phase transformation $\sigma_{s, T_{0}}^{M A}$. $h$ indicates the hardening modulus, and $r$ is the isotropic hardening function. The experimental results show that the stress increase caused by the phase transformation strain can be considered as linear hardening, and the gradient value $h$ is a constant parameter which can be expressed as follows:

— forward phase transformation

$$
h=h_{A M}=\frac{\sigma_{f, T}^{A M}-\sigma_{s, T}^{A M}}{\varepsilon_{L}} \quad \Delta \xi>0
$$


- reverse phase transformation

$$
h=h_{M A}=\frac{\sigma_{s, T}^{M A}-\sigma_{f, T}^{M A}}{\varepsilon_{L}} \quad \Delta \xi<0
$$

For linear hardening $r=h \varepsilon_{L} \xi$. Therefore, $\partial r / \partial \xi=h \varepsilon_{L}$. From the experimental results, it is found that the start stress of phase transformation with temperature is basically linear, which dose not consider the influence of plasticity on the final stress of the austenite reverse transformation for simplicity. Therefore, the evolution of transformation stress with temperature can be described with the use of the following equation

$$
\begin{array}{ll}
\sigma_{s, T}^{M A}=\sigma_{s, T_{0}}^{M A}+\left(T-T_{0}\right) C_{A M} & \sigma_{f, T}^{M A}=\sigma_{f, T_{0}}^{M A}+\left(T-T_{0}\right) C_{A M} \\
\sigma_{s, T}^{A M}=\sigma_{s, T_{0}}^{A M}+\left(T-T_{0}\right) C_{M A} & \sigma_{f, T}^{A M}=\sigma_{f, T_{0}}^{A M}+\left(T-T_{0}\right) C_{M A}
\end{array}
$$

in which $C_{A M}$ and $C_{M A}$ are material parameters which depict the slope of the phase transformation stress with temperature.

\subsection{Plastic strain evolution rule}

After finishing the stress-induced phase transformation, if the loading is continued up to exceed the martensite plastic yield stress, the plastic deformation of martensite will occur. As temperature is increased, the initial stress of the forward phase transformation increases. As the martensitic start stress is higher than the austenite plastic yield stress, the SMA will begin to undergo the austenite plastic deformation firstly. In this paper, it is assumed that the plastic yield behavior of austenite and martensite is subject to the von Mises yield criterion, and the plastic yield condition can be expressed as:

— austenitic plastic yield

$$
F_{A p}(\boldsymbol{\sigma}, p)=\sigma_{e q}-\sigma_{y}^{A}(p)=0
$$

— martensite plastic yield

$$
F_{M p}(\boldsymbol{\sigma}, p)=\sigma_{e q}-\sigma_{y}^{M}(p)=0
$$

in which the internal variable $p$ is the cumulative plastic strain

$$
\dot{p}=\left[\frac{2}{3} \dot{\varepsilon}_{p}: \dot{\varepsilon}_{p}\right]^{\frac{1}{2}}
$$

and $\sigma_{y}^{A}=\sigma_{y_{0}}^{A}+h_{A}^{p} p$ and $\sigma_{y}^{M}=\sigma_{y_{0}}^{M}+h_{M}^{p} p$ are the plastic yield stress of austenite and martensite, respectively. $\sigma_{y_{0}}^{A}$ and $\sigma_{y_{0}}^{M}$ are the initial plastic yield stress of austenite and martensite, respectively, and $h_{M}^{p}$ are the plastic yield modulus of austenite and martensite, respectively.

The plastic strain rate can be expressed as

$$
\dot{\varepsilon}_{p}= \begin{cases}\dot{\varepsilon}_{p}^{A}=\dot{\lambda}_{A} \frac{\partial F_{A p}(\boldsymbol{\sigma}, \xi)}{\partial \boldsymbol{\sigma}}=\sqrt{\frac{3}{2}} \dot{p} \mathbf{n}_{A} & \text { austenitic plastic yield } \\ \dot{\boldsymbol{\varepsilon}}_{p}^{M}=\dot{\lambda}_{M} \frac{\partial F_{M p}(\boldsymbol{\sigma}, \xi)}{\partial \boldsymbol{\sigma}}=\sqrt{\frac{3}{2}} \dot{p} \mathbf{n}_{M} & \text { martensite plastic yield }\end{cases}
$$

where $\lambda_{A}$ and $\lambda_{M}$ are plastic multipliers of the austenitic and martensite plastic yield, respectively. $\mathbf{n}_{A}$ and $\mathbf{n}_{M}$ are the direction vectors of the austenitic and martensite plastic yield, respectively

$$
\mathbf{n}_{A}=\mathbf{n}_{M}=\sqrt{\frac{3}{2}} \frac{\mathbf{s}}{\sigma_{e q}}
$$


The solutions for a plastic strain increment and the renewal of plastic strain are considered in the following. The plastic strain includes plastic strain of austenite and martensite. The course of the implicit stress integration is similar for both of them.

The implicit stress integral of plastic yield behavior can be solved by the method discussed in Section 2.3 for the phase transformation, which is similar to the equation of the deviatoric stress increment in Eq. (2.17)

$$
\mathbf{s}=\mathbf{s}^{*}-3 G \Delta \bar{\varepsilon}_{p} \frac{\mathbf{s}}{\sigma_{e q}}
$$

in which $\bar{\varepsilon}_{p}$ is the equivalent phase transformation strain. We take the contracted tensor product of each side of Eq. (2.33) with itself

$$
\sigma_{e q}+3 G \Delta p=\sigma_{e q}^{*}
$$

The expression form of the current equivalent plastic strain increment can be obtained by using the Newton-Raphson iteration method

$$
\mathrm{d} \Delta p=\frac{\sigma_{e q}^{*}-3 G \Delta p-r-\sigma_{y}}{3 G+h}
$$

In the above equations, $\sigma_{y}$ is the plastic yield stress of austenite $\sigma_{y}^{A}$ or martensite $\sigma_{y}^{M} . h$ is the plastic hardening modulus, which is the stress increment for the unit equivalent plastic strain increment. This modulus is also expressed as a linear hardening function. It means that the gradient value $h$ is constant, the expression for it is as follows

$$
h= \begin{cases}h_{A}^{p} & \text { austenitic plastic hardening modulus } \\ h_{M}^{p} & \text { martensitic plastic modulus of hardening }\end{cases}
$$

\subsection{Transformation initial conditions}

In many constitutive laws, researchers use stress tensors and temperature data as control variables. In this work, an effective criterion proposed in (Ben Jaber et al., 2008) using strain and temperature as control variables is used to judge the phase transformation points in order to avoid the complexity of transformation correction. To start the transformation, the following conditions should be satisfied.

The conditions for $A-M$ transformation are

$$
\varepsilon_{s}^{A M}<\bar{\varepsilon}_{t}^{A M}<\varepsilon_{f}^{A M} \quad \dot{\varepsilon}_{t}^{A M}>0 \quad 0<\xi<1 \quad \sigma_{e q}>\sigma_{s, T}^{A M}
$$

The $M-A$ transformation conditions are expressed as follows

$$
\varepsilon_{s}^{M A}<\bar{\varepsilon}_{t}^{M A}<\varepsilon_{f}^{M A} \quad \dot{\varepsilon}_{t}^{M A}<0 \quad 0<\xi<1 \quad \sigma_{e q}<\sigma_{s, T}^{M A}
$$

in which $\bar{\varepsilon}_{t}^{A M}$ and $\bar{\varepsilon}_{t}^{M A}$ are the equivalent phase transformation strain in $A$ - $M$ stage and $M-A$ stage, respectively.

The parameters of the initial and final strain for this transformation criterion are deduced from those of the stress phase transformation as follows.

The strain $\varepsilon_{s}^{A M}$ marking the beginning of the transformation band $A-M$

$$
\varepsilon_{s}^{A M}=\left\{\begin{array}{lll}
\varepsilon_{s c r}^{A M}+C_{A M}^{*}\left(T-T_{s}^{A M}\right) & \text { if } & T_{0}>T_{s}^{A M} \\
\varepsilon_{s c r}^{A M} & \text { if } & T_{0}<T_{s}^{A M}
\end{array}\right.
$$


The strain $\varepsilon_{f}^{A M}$ marking the end of the transformation band $A-M$

$$
\varepsilon_{f}^{A M}= \begin{cases}\varepsilon_{f c r}^{A M}+C_{A M}^{*}\left(T-T_{s}^{A M}\right) & \text { if } T_{0}>T_{s}^{A M} \\ \varepsilon_{f c r}^{A M} \text { if } & T_{0}<T_{s}^{A M}\end{cases}
$$

in which $C_{A M}^{*}=C_{A M} / E_{M}, \varepsilon_{s c r}^{A M}=\sigma_{s, T_{0}}^{A M} / E_{A}, \varepsilon_{f c r}^{A M}=\sigma_{f, T_{0}}^{A M} / E_{M}+\varepsilon_{L}$, and $T_{0}$ denotes the specified temperatures, i.e., room temperature.

It should be noted that $E_{A}$ and $E_{M}$ are temperature-dependent elastic moduli of austenite and martensite, which can be expressed by the following equation at a certain temperature

$$
\begin{aligned}
& E_{A}=E_{A}^{T_{0}}+k E_{A}^{T_{0}} \ln \left(T-T_{0}+1\right) \\
& E_{M}=E_{M}^{T_{0}}+k E_{M}^{T_{0}} \ln \left(T-T_{0}+1\right)
\end{aligned}
$$

where $E_{A}^{T_{0}}$ and $E_{M}^{T_{0}}$ are the elastic moduli of austenite and martensite at specified temperatures, i.e., room temperature. $k$ is a material parameter which depicts the increasing rate of the elastic modulus with temperature and is obtained from experimental results.

The state functions of the beginning strain $\varepsilon_{s}^{M A}$ and the end strain $\varepsilon_{f}^{M A}$ of the transformation band $M-A$ are

$$
\varepsilon_{s}^{M A}=C_{M A}^{*}\left(T-T_{s}^{M A}\right)+\varepsilon_{L} \quad \varepsilon_{f}^{M A}=C_{M A}^{*}\left(T-T_{f}^{M A}\right)
$$

in which $C_{M A}^{*}=C_{M A} / E_{A}$.

\section{Finite element implementation}

\subsection{Consistent tangent modulus of phase transformation}

After differentiation of Eqs. (2.24), it can be derived

$$
\begin{aligned}
& \left(1+3 G \frac{\varepsilon_{L} \Delta \xi}{\sigma_{e q}}\right) \delta \mathbf{s}-\frac{3 G \varepsilon_{L}}{\sigma_{e q}} \delta \Delta \xi \mathbf{s}-\frac{3 G \varepsilon_{L} \Delta \xi}{\sigma_{e q}^{2}} \delta \sigma_{e q} \mathbf{s}=\delta \mathbf{s}^{*} \\
& \delta \sigma_{e q}+3 G \varepsilon_{L} \delta \Delta \xi=\delta \sigma_{e q}^{*}
\end{aligned}
$$

To differentiate Eq. (2.10), we put $\delta F=\delta \sigma_{e q}-\delta r=0$. Therefore, it can be obtained $\delta \sigma_{e q}=\delta r=h \varepsilon_{L} \delta \xi=h \varepsilon_{L} \delta \Delta \xi$. So, we may write Eq. (3.1) 2 as

$$
h \varepsilon_{L} \delta \Delta \xi+3 G \varepsilon_{L} \delta \Delta \xi=\delta \sigma_{e q}^{*} \quad \text { or } \quad \delta \Delta \xi=\frac{\delta \sigma_{e q}^{*}}{(h+3 G) \varepsilon_{L}}
$$

Combining with $(3.1)_{2}$ it gives

$$
\delta \sigma_{e q}=\delta \sigma_{e q}^{*}\left(1-\frac{3 G}{h+3 G}\right)
$$

We now use Eqs. $(3.2)_{2}$ in Eq. (3.1) 1 to eliminate $\delta \sigma_{e q}, \delta \Delta \xi$ and $\Delta \xi$, respectively, to give

$$
\frac{\sigma_{e q}^{*}}{\sigma_{e q}} \delta \mathbf{s}+\frac{\delta \sigma_{e q}^{*}}{\sigma_{e q} \sigma_{e q}}\left(\sigma_{e q}-\frac{3 G \sigma_{e q}^{*}}{h+3 G}\right) \mathbf{s}=\delta \mathbf{s}^{*}
$$

Consider the term

$$
\delta \sigma_{e q}^{*}=\frac{3}{2} \frac{1}{\sigma_{e q}^{*}} \mathbf{s}^{*}: \delta \mathbf{s}^{*}
$$


Substituting this together with the expression for $\mathbf{s}$ in Eq. (2.22) into Eq. (3.4) gives

$$
\delta \mathbf{s}=\frac{3}{2}\left(\frac{\mathbf{s}^{*}}{\sigma_{e q}^{*}} \otimes \frac{\mathbf{s}^{*}}{\sigma_{e q}^{*}}\right)\left(\frac{3 G}{h+3 G}-\frac{\sigma_{e q}}{\sigma_{e q}^{*}}\right): \delta \mathbf{s}^{*}+\frac{\sigma_{e q}}{\sigma_{e q}^{*}} \delta \mathbf{s}^{*}
$$

If we write

$$
Q=\frac{3}{2}\left(\frac{3 G}{h+3 G}-\frac{\sigma_{e q}}{\sigma_{e q}^{*}}\right) \quad R=\frac{\sigma_{e q}}{\sigma_{e q}^{*}}
$$

then Eq. (3.6) becomes

$$
\delta \mathbf{s}=\left[Q\left(\frac{\mathbf{s}^{*}}{\sigma_{e q}^{*}} \otimes \frac{\mathbf{s}^{*}}{\sigma_{e q}^{*}}\right)+R \mathbf{I}\right]: \delta \mathbf{s}^{*}
$$

in which $\mathbf{I}$ is the fourth-order identity tensor.

We are trying to relate $\delta \mathbf{s}$ and $\delta \boldsymbol{\varepsilon}$ to derive the Jacobian. The deviatoric trial stress in terms of the deviatoric strain may be written as follows with applying the differential operator

$$
\delta \mathbf{s}^{*}=2 G\left(\delta \boldsymbol{\varepsilon}-\frac{1}{3} \mathbf{I} \otimes \mathbf{I}: \delta \boldsymbol{\varepsilon}\right)
$$

Substituting into Eq. (3.7) gives

$$
\begin{aligned}
\delta \mathbf{s}^{*} & =2 G\left[Q\left(\frac{\mathbf{s}^{*}}{\sigma_{e q}^{*}} \otimes \frac{\mathbf{s}^{*}}{\sigma_{e q}^{*}}\right)+R \mathbf{I}\right]:\left[\delta \varepsilon-\frac{1}{3}(\mathbf{I} \otimes \mathbf{I}): \delta \varepsilon\right]=2 G Q\left(\frac{\mathbf{s}^{*}}{\sigma_{e q}^{*}} \otimes \frac{\mathbf{s}^{*}}{\sigma_{e q}^{*}}\right): \delta \varepsilon \\
& +2 G R \delta \varepsilon-\frac{1}{3} Q\left(\frac{\mathbf{s}^{*}}{\sigma_{e q}^{*}} \otimes \frac{\mathbf{s}^{*}}{\sigma_{e q}^{*}}\right):[(\mathbf{I} \otimes \mathbf{I}): \delta \boldsymbol{\varepsilon}]-\frac{2}{3} G R \mathbf{I}:[(\mathbf{I} \otimes \mathbf{I}): \delta \varepsilon]
\end{aligned}
$$

The stress can be given in terms of its deviatoric tensor as

$$
\delta \boldsymbol{\sigma}=\delta \mathbf{s}+\frac{1}{3}(\mathbf{I} \otimes \mathbf{I}): \delta \boldsymbol{\sigma}=\delta \mathbf{s}+K(\mathbf{I} \otimes \mathbf{I}): \delta \varepsilon
$$

in which $K=E /[3(1-2 \nu)]$ is the bulk modulus.

Substituting into (3.9) gives

$$
\frac{\delta \boldsymbol{\sigma}}{\delta \varepsilon}=2 G Q\left(\frac{\mathbf{s}^{*}}{\sigma_{e q}^{*}} \otimes \frac{\mathbf{s}^{*}}{\sigma_{e q}^{*}}\right)+2 G R \mathbf{I}+\left(K-\frac{2}{3} G R\right)(\mathbf{I} \otimes \mathbf{I})
$$

This is the consistent tangential modulus for the forward and reverse phase transformation.

\subsection{Consistent tangent modulus of plastic deformation}

The solving process for the consistent tangent modulus of the plastic strain behavior of the NiTi alloy is similar to that of the previous phase transformation behavior. By differentiating Eq. (2.33), we obtain

$$
\begin{aligned}
& \left(1+3 G \frac{\Delta p}{\sigma_{e q}}\right) \delta \mathbf{s}-\frac{3 G \delta \Delta p}{\sigma_{e q}} \mathbf{s}-\frac{3 G \Delta p}{\sigma_{e q}^{2}} \delta \sigma_{e q} \mathbf{s}=\delta \mathbf{s}^{*} \\
& \delta \sigma_{e q}+3 G \delta \Delta p=\delta \sigma_{e q}^{*}
\end{aligned}
$$

and $\delta \sigma_{e q}=\delta r=h \delta p=h \delta \Delta p$.

The consistent tangent modulus expression of the plastic strain behavior is the same as Eq. $(3.12)_{2}$. The difference from the phase transformation strain behavior is the expression of the plastic hardening modulus $h$. A reverse mapping algorithm is described in Algorithm 1. 
Algorithm 1: Iteration procedure

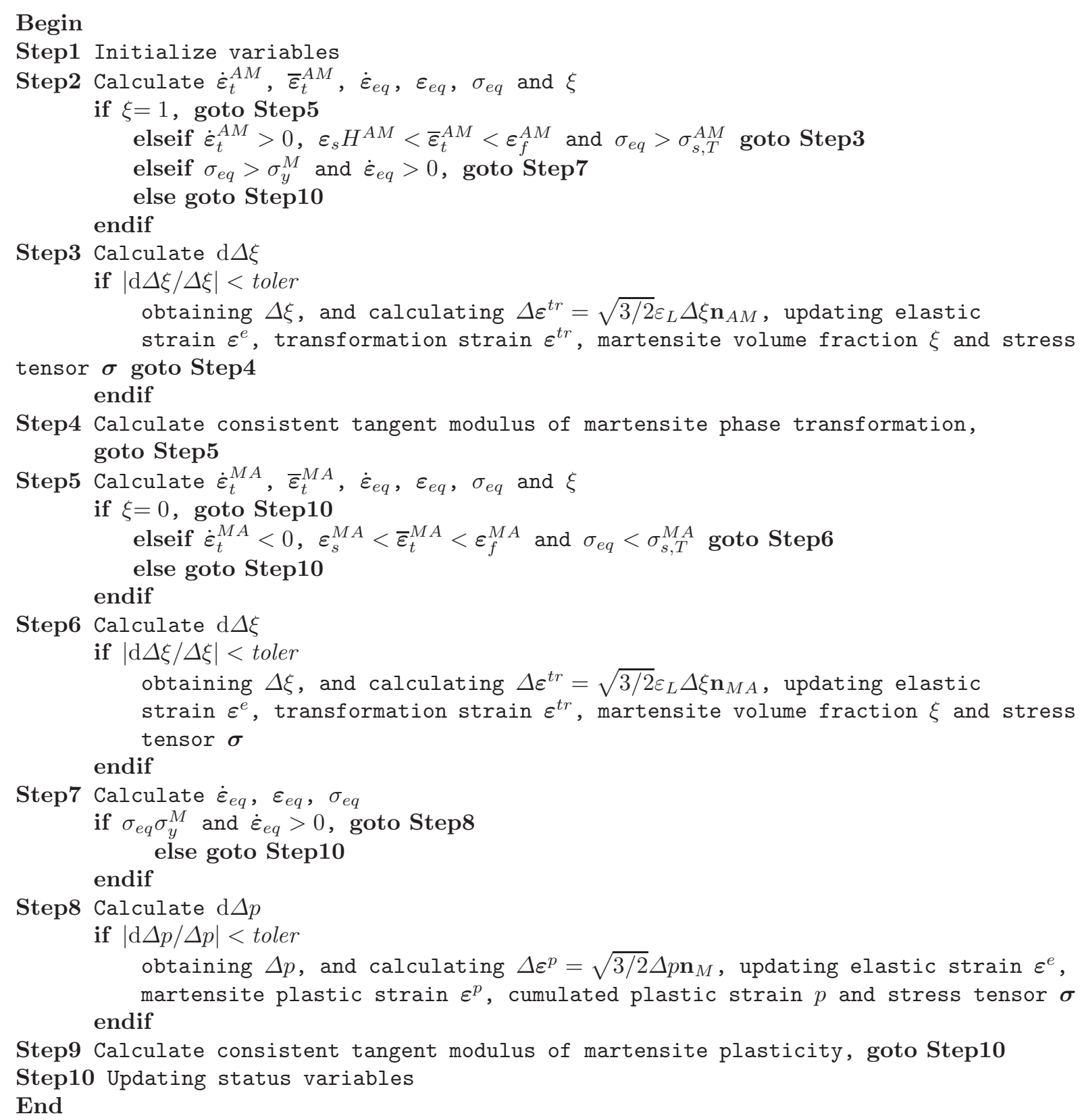

\section{Verification of the proposed model}

The constitutive model outlined in Section 2 has been implemented as a user subroutine for the finite element code ANSYS (2004) to analyze the phase transformation and plastic deformation under the uniaxial tension and unloading condition. In the following, the results of simulations by the proposed model are compared with the experimental data by Kang et al. (2009). From the experimental stress-strain curve under different temperatures, the following material parameters can be used. A SMA phase transformation and plasticity behavior diagram is presented in Fig. 1 to identify the parameters: $E_{A}^{T_{0}}=41.0 \mathrm{GPa}, E_{M}^{T_{0}}=37.0 \mathrm{GPa} ; v_{A}=v_{M}=0.33$; $C_{A M}=8.0 \mathrm{MPa} / \mathrm{K}, C_{M A}=8.8 \mathrm{MPa} / \mathrm{K} ; k=0.16 ; \sigma_{s, T 0}^{A M}=53.0 \mathrm{MPa}, \sigma_{f, T 0}^{A M}=381.0 \mathrm{MPa}$, $\sigma_{s, T 0}^{M A}=141.0 \mathrm{MPa}, \sigma_{f, T 0}^{M A}=122.0 \mathrm{MPa} ; \varepsilon_{L}=0.035 ; T_{0}=295 \mathrm{~K} ; h_{A M}=0.8 \mathrm{GPa} ;$ $h_{M A}=1.3 \mathrm{GPa} ; h_{M}^{p}=6.7 \mathrm{GPa} ; \sigma_{y}^{M}=1.7 \mathrm{GPa}$. 


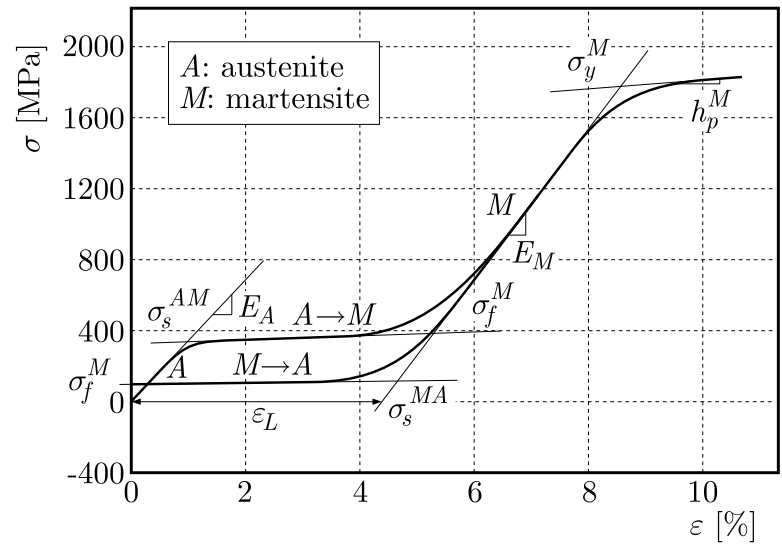

Fig. 1. SMA phase transformation and plasticity behavior diagram

(a)

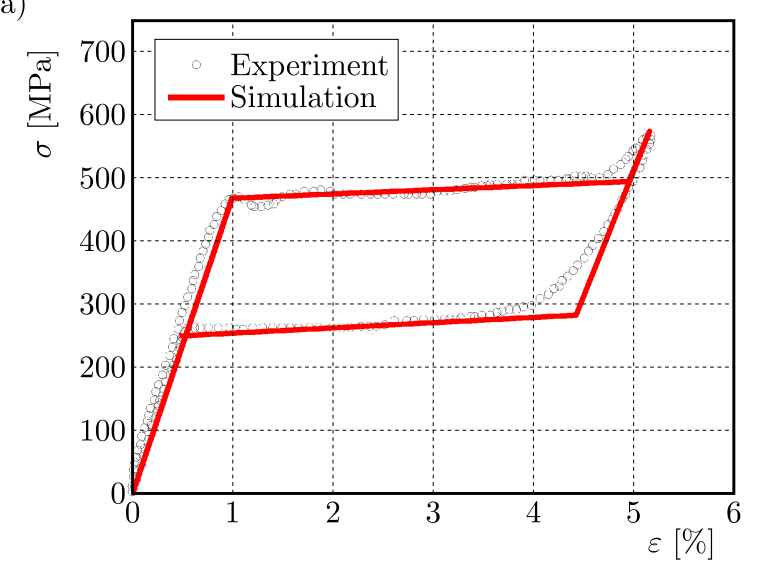

(c)

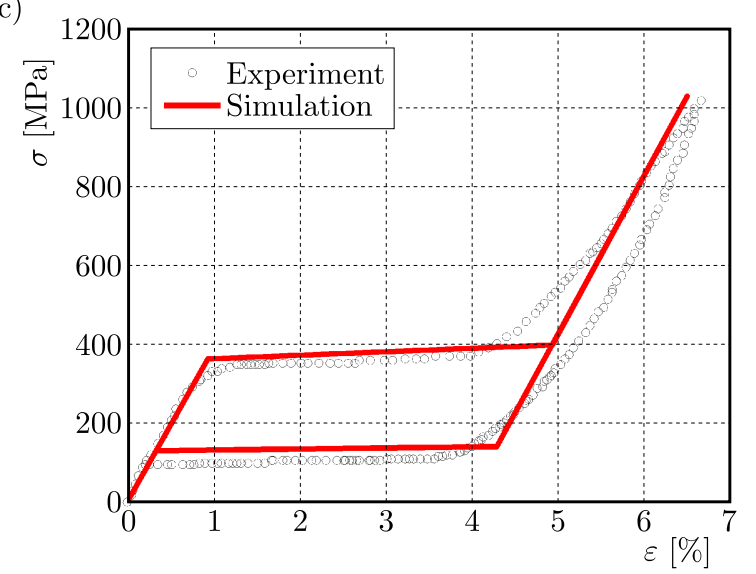

(b)

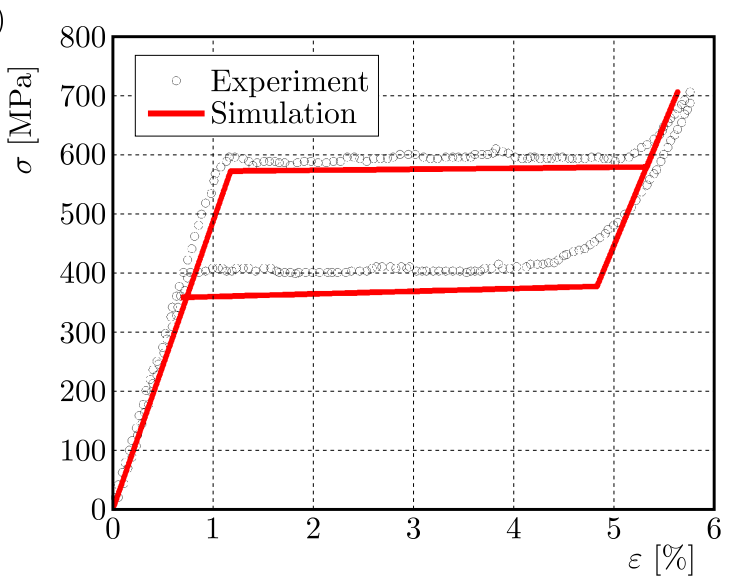

(d)

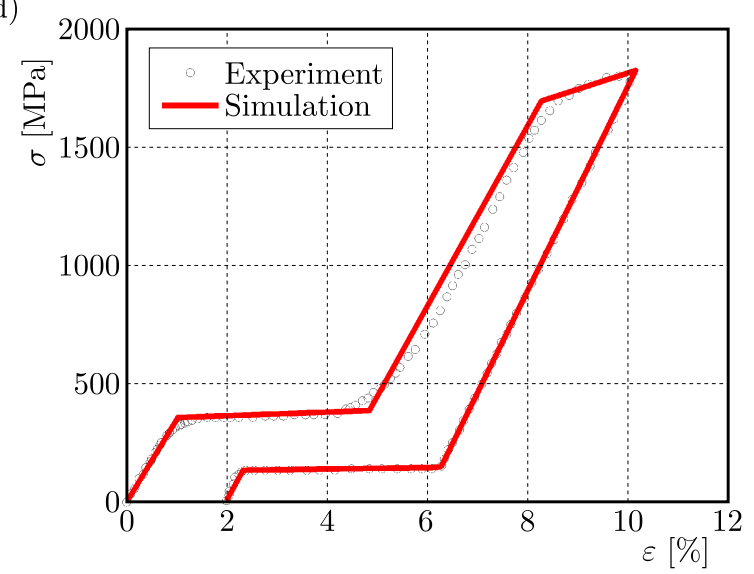

Fig. 2. Stress-strain curves during tension and unloading at various temperatures under strain controlled loading: (a) $309 \mathrm{~K}$, (b) $323 \mathrm{~K}$, (c) $295 \mathrm{~K}$, peak strains at $6.6 \%$, (d) $295 \mathrm{~K}$, peak strains at $10 \%$

The uniaxial tension and unloading case of the material has been calculated using ANSYS based on the proposed model and employed a 3D 8-node iso-parametric brick element. The simulation results are shown in Fig. 2. It can be seen that the stress-induced martensitic transformation occurs at low temperatures, such as $295 \mathrm{~K}$, when the stress is applied to the forward phase transformation stress $\sigma_{s, T}^{A M}$. When the stress reaches the end of the forward phase transformation $\sigma_{f, T}^{A M}$, the phase transformation strain can reach the maximum phase transformation strain $\varepsilon_{L}$, and the martensitic transformation is over. During unloading, once the stress reaches 
the initial stress $\sigma_{s, T}^{M A}$ of martensite to austenite, the martensite gradually transforms to austenite. As the load is lower than the phase transformation final stress $\sigma_{f, T}^{M A}$, the elastic unloading of austenite takes place in the aftermath. It can be seen in Fig. 2 that the phase transformation stress is increased with an increase in temperature. The elastic modulus also increases with the increase in temperature.

Figures 2c,d show the stress-strain curves obtained in the tension-unloading with high peak strains at $295 \mathrm{~K}$. It can be seen in Fig. 2c that the response peak stress is about $1000 \mathrm{MPa}$, and the martensite can still be completely transformed into austenite when undergoing unloading. However, with a higher applied peak strain, an apparent residual strain can be observed which is caused by the plastic deformation of martensite, as shown in Fig. 2d. The simulated results show a good agreement with the experimental ones. The results show that the proposed constitutive model can predict the thermodynamic behavior in the super-elastic NiTi alloy. It can be predicted that the model can also give reasonable prediction results for other working conditions and other experimental results in the temperature range.

Figure 3 shows a finite element model of an SMA rod with multiple elements, with length of $10 \mathrm{~mm}$ and radius of $0.5 \mathrm{~mm}$. The fixed boundary conditions are applied at one end, at the other side $\Delta l=0.1 \mathrm{~mm}, 0.3 \mathrm{~mm}, 0.5 \mathrm{~mm}$ and $0.7 \mathrm{~mm}$ (direction along the $Z$ axis), and then a total torque load $T_{M}=25 \mathrm{Nm}$ is applied to the peripheral node of this side at $T_{0}=295 \mathrm{~K}$. The stress-strain curves in the tension-torque loading is calculated based on the data at the Gauss point of the picked element. It can be seen in Fig. 3a that the tension stress exhibits three inflection points. One is the phase transformation starting point $A$, another one is the phase transformation ending point $B$, and last one is the yield point $C$. It can be concluded that the phase transformation and plasticity lead to a decrease in the tension stress for the exact tension strain value. From Fig. 3b it can be found that as the tension strain is small, the tension rod dose not go enough through the phase transformation in the tension stage, such as in the $T_{M}=25 \mathrm{Nm}$ and $\Delta l=0.1 \mathrm{~mm}$ or $\varepsilon=0.01$ case. As the total torque load continues to be applied, the tension rod would go through the phase transformation and martensite plasticity stages, which implies the non-homogeneous stress status including both tensile and shear stresses. When the tension strain is high enough, such as in the $T_{M}=25 \mathrm{Nm}$ and $\Delta l=0.7 \mathrm{~mm}$ or $\varepsilon=0.07$ case, the tension rod finishes phase transformation in this stage. Then, as the total torque load is applied enough high, the tension rod would just go through the martensite plasticity stage. In this case, the plastic transform point mapped by the shear stress shown in Fig. 3b presents a decrease as the tension strain increases.

(a)

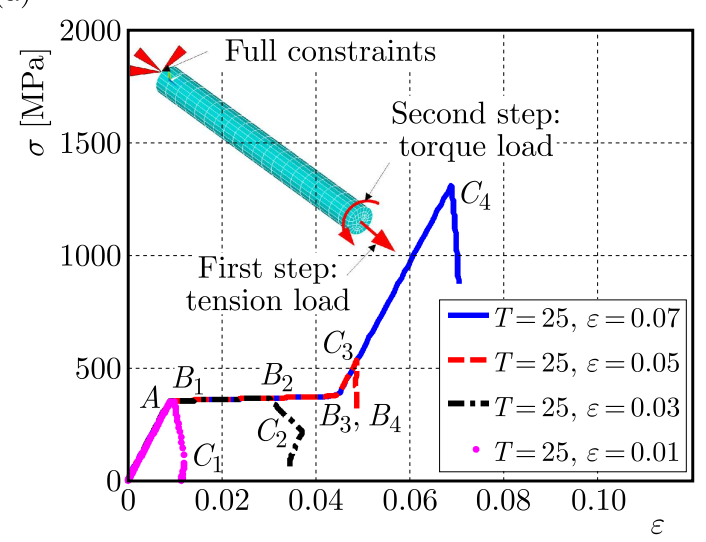

(b)

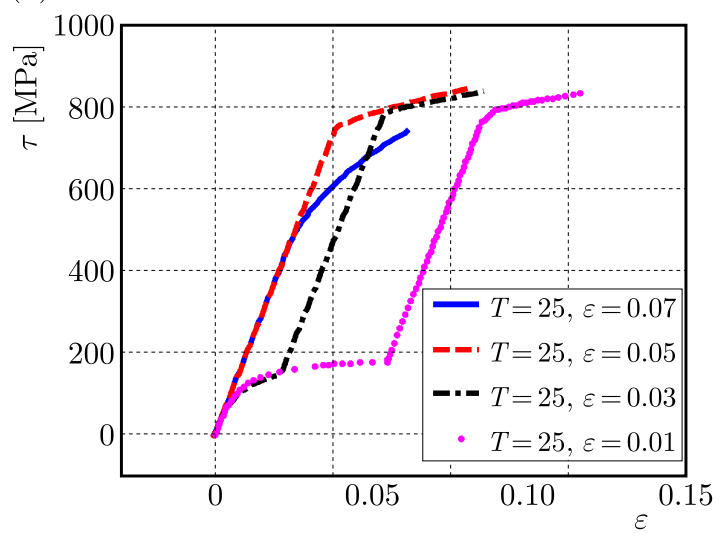

Fig. 3. Finite element analysis of the SMA rod with multiple elements: (a) tension stress, (b) shear stress 


\section{Conclusions}

A shape memory alloy (SMA) phenomenological constitutive model is proposed in the general inelastic framework. Transformation hardening, reverse transformation, temperature dependence of transformation strain for each phase and plasticity hardening are considered in the proposed model. The model is implemented into the finite element code ANSYS by USERMAT user subroutine. The numerical results show that good simulation results can be obtained for uniaxial tensile-unloading stress-strain curves. The simulation results of the proposed model show a good agreement with the experimental ones at different temperatures for the super-elastic behavior of NiTi alloy. Finally, the accuracy of the implementation is verified by numerical tests considering non-homogeneous deformation of the structure.

\section{Acknowledgments}

We would like to thank for the financial support of this work by the National Natural Science Foundation of China (NSFC) under Grant Number 51775406 and 51405371, Open Research Fund of State Key Laboratory for Manufacturing Systems Engineering (Grant No. sklms2016007), Open Research Fund of State Key Laboratory of Structural Analysis for Industrial Equipment (Grant No. GZ1612), 111 Project B14042, Fundamental Research Funds for the Central Universities (Grant No. JB160403), Natural Science Foundation of Shanxi Province of China (Grant No. 2017JM5035), the Natural Science Foundation of Guangxi Province of China (Grant No. 2016GXNSFBA380230), and Natural Science Foundation of Hunan Province of China (Grant No. 2015JJ2054).

\section{References}

1. ANSYS Structural Analysis Guide, 2004, Version 8.0

2. Auricchio F., TAYlor R.L., 1997, Shape-memory alloy modeling and numerical simulations of the finite-strain superelastic behavior, Computer Methods of Application Mechanics and Engineering, 143, 175-194

3. Ben Jaber M., Smaoui H., Terriault P., 2008, Finite element analysis of a shape memory alloy three-dimensional beam based on a finite strain description, Smart Materials and Structures, $\mathbf{1 7}, 4,5202-5228$

4. Bo Z., LaGoudas D.C., 1999, Thermomechanical modeling of polycrystalline SMAs under cyclic loading, Part I: theoretical derivations, International Journal of Engineering Science, 37, 1089-1140

5. Bouvet C., Calloch S., Lexcellent C., 2004, A phenomenological model for pseudoelasticity of shape memory alloys under multi-axial proportional and nonproportional loadings, European Journal of Mechanics A-Solids, 23, 37-61

6. Cisse C., Zaki W., Zineb T. B., 2016, A review of constitutive models and modeling techniques for shape memory alloys, International Journal of Plasticity, 76, 244-284

7. KAN Q.H., Kang G.Z., QIAN L.M., 2010, Super-elastic constitutive model considering plasticity and its finite element implementation, Acta Mechanica Solida Sinica, 23, 2, 95-105

8. KANG G.Z., KAN Q.H., QIAN L.M., LiU Y.J., 2009, Ratchetting deformation of super-elastic and shape-memory NiTi alloys, Mechanics of Materials, 41, 139-153

9. Lagoudas D.C., Bo Z., Qidwai, M.A., 1996, A unified thermodynamic constitutive model for SMA and finite element analysis of active metal matrix composites, Mechanics of Composite Materials and Structures, 3, 2, 153-179

10. LazGhab T., 2001, Modeling of shape memory alloys with plasticity, Ph.D. Thesis, Florida International University, Florida

11. Liang C., Rogers C., 1990. One-dimensional thermomechanical constitutive relations for shape memory materials, Journal of Intelligent Material Systems and Structures, 1, 207-234 
12. Lubliner J., Auricchio F., 1996, Generalized plasticity and shape memory alloys, International Journal of Solids and Structures, 33, 991-1003

13. McKelvey A.L., Ritchie R.O., 2001, Fatigue-crack growth behavior in the superelastic and shape-memory alloy Nitinol, Metallurgical and Materials Transactions A, 32, 3, 731-743

14. Saint S., Chirani S.A., Calloeh S., 2009. A 3D super-elastic model for shape memory alloys taking into account Progressive strain under cyclic loadings, Mechanics of Materials, 41, 1, 12-26

15. TANAKA T., 1986, A thermomechanical sketch of shape memory effect: one dimensional tensile behavior, Res Mechanica, 18, 251-263

16. Yan W., Wang C.H., Zhang X.P., Mai Y.W., 2003, Theoretical modelling of the effect of plasticity on reverse transformation in superelastic shape memory alloys, Materials Science and Engineering A, 354, 1, 146-157

Manuscript received August 4, 2016; accepted for print June 24, 2017 